\title{
(Research Article) \\ Comparison of Transform Techniques Based Image Fusion for Effective Disease Diagnosis
}

\author{
Dr.T.R. Ganesh Babu ${ }^{1 *}$, Dr.J. Kirubakaran ${ }^{2}$, R. Dhivyabharathi ${ }^{3}$ \\ ${ }^{1 *}$ Department of ECE, Muthayammal Engineering College,Namakkal,Tamil Nadu, INDIA \\ ${ }^{2}$ Department of Electronics and Communication Engineering, Muthayammal Engineering College,Namakkal,Tamil Nadu, INDIA \\ ${ }^{3}$ Master of Biomedical engineering, Anna University, Chennai,Tamil Nadu, INDIA
}

\begin{abstract}
Medical image fusion is pivotal to extract accurate information from medical images for disease diagnosis. This is used to amalgamate various images from same or different sources. In this work, Computed Tomography (CT) and Positron Emission Tomography (PET) images are fused by means of Discrete Wavelet Transform (DWT), curve let transform and multimodal image fusion. The current researches for disease diagnosis mainly focus on the identification of optimal method that consumes less time with high accuracy in order to ensure patient safety. Therefore, this work aims to find the suitable method by comparing various transform techniques for accurate analysis of medical images. The CT and PET images are used in this paper to obtain both anatomical and physiological information of human body and these images are utilized for image fusion by principal component analysis (PCA) and maximum method. The reliability of the techniques is calculated by performance analysis and is hence inferred that multimodal image fusion provides better accuracy than the rest of the methods. However, the future work will be to validate the results for a greater number of input images.
\end{abstract}

Keywords: image fusion, CT and PET, discrete wavelet transform, curvelet transform, multimodal image fusion, PCA, maximum method.

\section{Introduction}

Image fusion is widely used in medical field for assessing different health issues such as Alzheimer's disease and cancer. There are many image fusion techniques [1] in existence and in this study PCA and simple maximum method are incorporated to combine CT and PET images. PCA is used as it lowers the noise level and also intensifies the features in the input image for observation. It comes under spatial domain and it reduce the size of input without any loss of information. Simple maximum method also comes under spatial domain and in this method maximum values of pixels are taken from the input image to form the fused image [2]. The fused image is helpful to perform image processing such as edge detection and segmentation in order to get the required information with better contrast.

The CT and PET images considered in this work as PET provides metabolic process details and CT presents cross sectional information for the diagnosis of various medical

*Corresponding Author: e-mail: ganeshbabutr@gamil.com, Tel-+91-9344985019

ISSN 2320-7590

(C) 2019 Darshan Institute of Engg. \& Tech., All rights reserved problems. Furthermore, the CT-PET image combination gives exact location of infected cyst among other noninfected parts which is helpful for accurate detection of cancer [3].

\section{Related Works}

Gattim et al, presented multimodal image fusion using curvelet and Genetic Algorithm [GA]. In this paper, Magnetic Resonance Imaging (MRI) and CT images are used and wavelet transform is applied before image fusion. It is compared with the combination of curvelet transform with GA. Later it is concluded that curvelet and GA possess better fusion characteristics than wavelet transform [4]. Radhika et al, introduced neuro fuzzy logic based fusion algorithm for multimodality images. This technique is employed to avoid information loss, false edge detection, dark spots in tissues and spatial distortion problem [5].

Gaurav et al, proposed directive contrasts based multimodal medical image fusion in Non-Subsampled Contourlet Transform (NSCT) domain. In this work, the source images are transformed into NSCT and then 
combining low and high frequency components. The fusion rules involved in this paper are based on phase congruency and directive contrast. The result shows that it gives an effective way for examining multimodal images [6]. Arthur et al, presented nonsubsampled contourlet transform theory, design and applications.

This paper is based on non-subsampled pyramid structure, nonsubsampled directional filter banks and ladder structure. It is applied for image denoising and enhancement applications [7]. Huda Ahamed et al, proposed medical image fusion algorithm based on local average energy motivated Pulse Coupled Neural Network (PCNN) in NSCT domain. The local average energy is to motivate the pulse coupled neural network in order to capture edges, contours and textures.

The advantage in this method is that it avoids contrast reduction, edge blurring and unwanted degradations [8]. Indira et al, introduced performance evaluation of DWT, SWT and NSCT for fusion of PET and CT images using different fusion rules. It is inferred that average fusion rule for low frequency coefficient and gradient fusion rule for high frequency coefficient give more desirable result than other fusion rules for DWT, Stationary Wavelet Transform (SWT) and NSCT. It is also observed that the time taken for the execution of SWT is higher than DWT and NSCT.

This paper then concluded that average and gradient fusion rules suit well for medical images [9]. Anjali et al, presented wavelet transform based medical image fusion with different fusion methods. It is declared that wavelet transform is appropriate for medical images by applying maximum, minimum and mean methods. The quality of the result is evaluated by means of entropy value [10].

\section{Methods}

This work is carried out by using MATLAB software and the preprocessing involves image registration, image resizing and histogram equalization. Image Registration is the process of combining images which assigns one image as reference image and the other as target image. Then the target image is aligned with the help of reference image. The image resizing is needed if the size of these images differs from one another. In addition, histogram equalization is also essential to enhance the contrast of the image. The algorithm for image fusion using DWT [11] includes re sampling and registering of the input images which are CT image and PET image. It is further processed by applying DWT to decompose it into four sub-bands (low -low [LL], low- high [LH], high-low [HL], high-high $[\mathrm{HH}])$. Thus, the wavelet coefficients obtained from both the images are called approximation coefficients, horizontal detail coefficients, vertical detail \& diagonal detail coefficients. The fusion rules such as PCA and maximum method are applied to these coefficients and hence fused wavelet coefficients are obtained.
Furthermore, inverse discrete wavelet transform [IDWT] is performed to get the fused image. Nevertheless, this technique fails to localize the curved edges and so curvelet transform is performed by using the fusion rules PCA and maximum method. The curvelet transform image fusion algorithm [12] first involves image registration. The four stages of curvelet transform are sub-band decomposition, smooth partitioning, renormalization and ridgelet analysis. The registered input images have to undergo these four stages in order to get the curvelet coefficients. The fusion rules such as PCA and maximum method are applied further. Finally, the fused image is obtained by performing Inverse Curvelet Transform [ICVT].

The PCA algorithm [13] includes column vector generation from input image and then covariance matrix is generated. The diagonal elements of the covariance matrix contain the variance of each column vector. In addition, eigen values and eigen vectors are calculated and normalize the column vector corresponding to larger eigen value by dividing each element with mean of eigen vector. The output values of the normalized eigen vector are multiplied with each pixel of the input images. The sum of these two matrices is the fused image matrix.

In maximum fusion rule, the higher magnitude complex wavelet coefficients replace the smaller magnitude complex wavelet coefficients. In the input images, the pixel with maximum intensity is chosen and selected to form the fused image.

The algorithm steps involve

If, LL1(i,j) > LL2(i,j) Lout(i,j) = LL1(i,j); else Lout( $(\mathrm{i}, \mathrm{j})=$ LL2(i,j);

Where, LL=indicates low frequency coefficients, Lout=indicates output image value, LL1=indicates coefficients of CT image and LL2=indicates coefficients of PET image.

The below block diagram represents the multimodal image fusion. The wavelet transform (frequency domain) is widely used for image processing applications. However, the main disadvantage of DWT is the limitation of shift invariance which is caused by up and down sampler in the design of wavelet filters.

Hence, a small change in the input signal causes serious variations in the wavelet coefficients. To overcome this disadvantage of DWT, the design of DWT is modified by just removing the up and down-sampler which is called Undecimated Wavelet Transform [UWT].

Fig. 1 (a) and (b) illustrate the block diagram of UWT and UWT decomposition filter design respectively. 


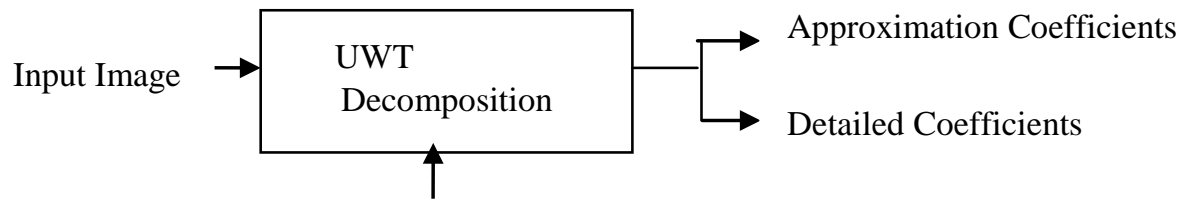

Level of Decomposition

Figure 1. (a) Block diagram of UWT

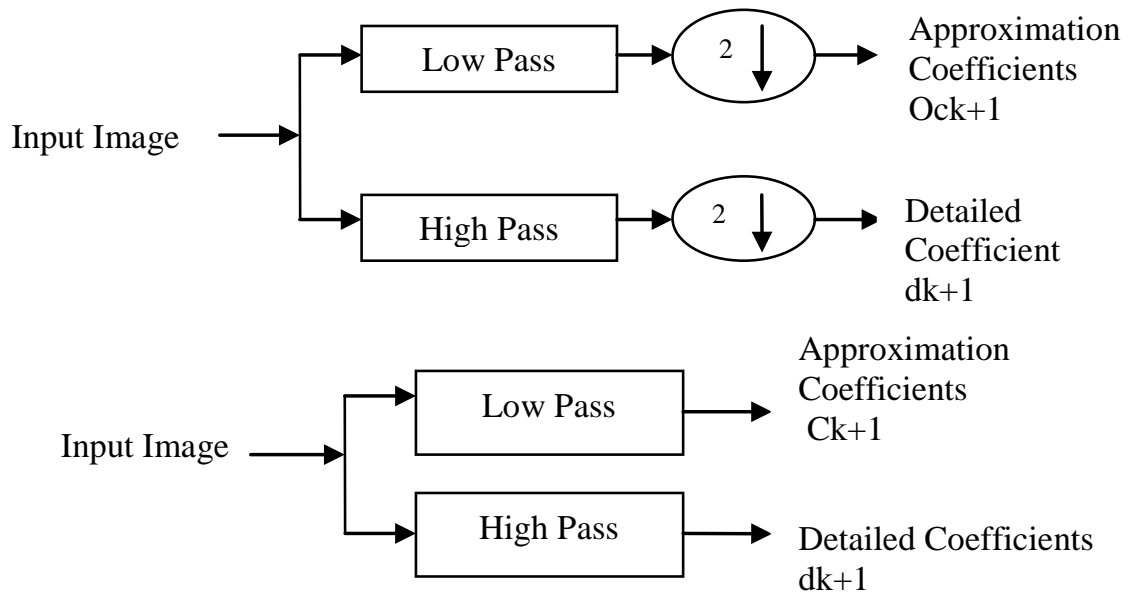

Figure 1. (b) Decomposition filter design UWT

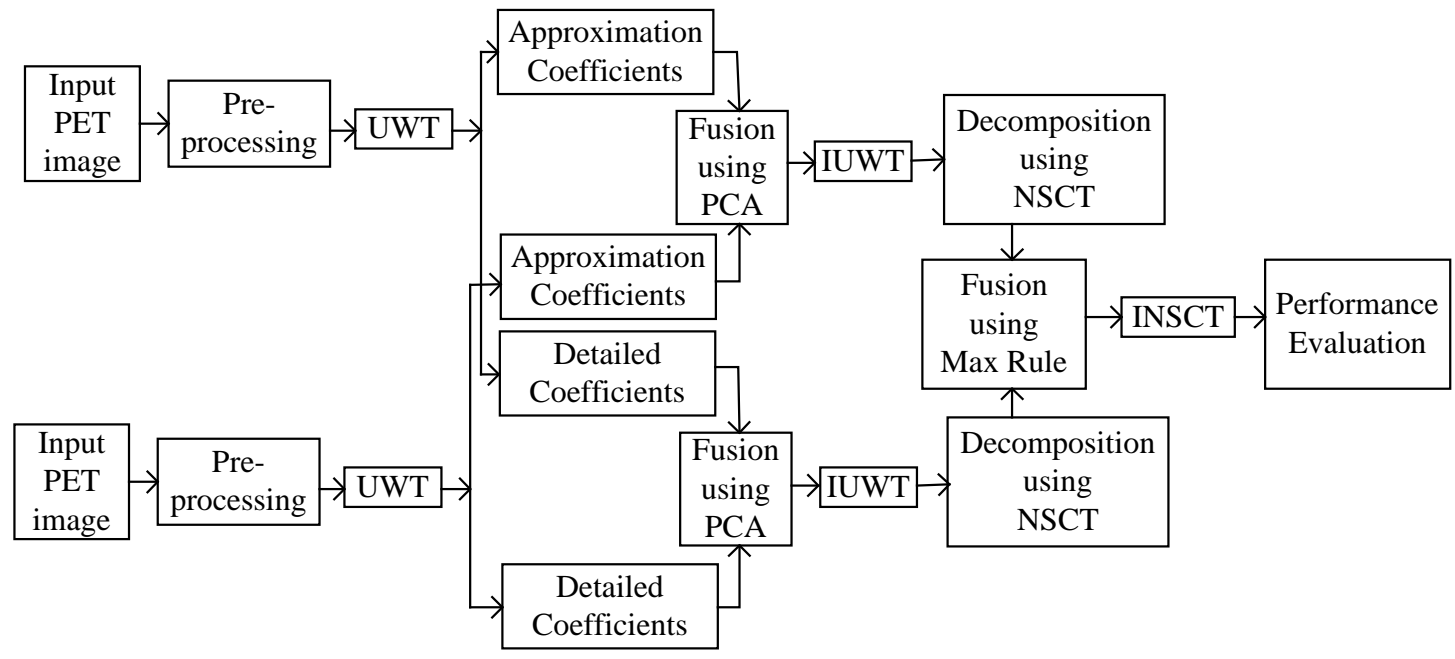

Figure 2. Multi model Image Fusion

The up and down samples are removed from the UWT filter design and hence, sub band size obtained from input image and UWT decomposition are same. UWT generates a high dimensional feature space than the DWT feature space and therefore an efficient feature selection algorithm is needed to reduce the dimensionality of the feature space. In decomposition stage1, fusion algorithm is applied in UWT domain. UWT [14] decomposes the preprocessed input images into approximation coefficients which are low frequency components and detailed coefficients which are high frequency components. The decomposed approximation and detailed coefficients from each of the input images are fused by PCA to improve the nondirectionality limitation of UWT. Then the fused images are reconstructed using the inverse UWT. The whole operations carried out in this stage serves to provide significant 
localization of features in the fused image. In decomposition stage 2, fusion algorithm is applied in NSCT domain. The NSCT has been proposed in order to overcome the disadvantage of shift variance introduced due to wavelets and to hold the directional and multi scale properties of the transform. It also decomposes the images in the form of contour segments. The NSCT does not occupy the downsampling as in UWT, hence it is unaffected by any shift in the input images. After applying NSCT, a set of coefficients are obtained for both the input images that represents Approximation (low frequency) and detailed (high frequency) coefficients. The obtained coefficients from NSCT are then fused using the maximum fusion rule. This fusion rule chooses the maximum valued coefficients among each set of low/high frequency coefficients from input images in order to improve the visual quality of the fused image. The fused coefficients are then reconstructed using the inverse NSCT transform. Finally, the performance of this technique is analyzed by performance metrics. The below block diagram shows the multimodal image fusion [15].

\section{Results}

The proposed techniques are tested by using 25 images obtained from Bharat Scans, Chennai, India. It is observed from the experimental results that the multimodal image fusion of CT and PET images provides better outcome for all the input dataset. The below Figure 3(a) and (b) show CT and PET image. Figure 3(c) and (d) show fused image using Wavelet transform based PCA and maximum fusion rule.

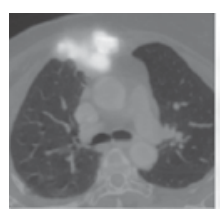

(a)

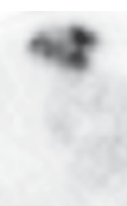

(b)

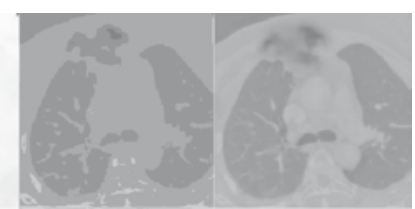

(c) (d)
Figure 3. Result of fused image using Wavelet Transform (a) CT Image (b) PET Image (c) PCA (d) Maximum method
The following Fig.4(a) and fig.4(b) shows CT and PET image. Fig.4(c) and fig.4 (d) shows fused image using Curvelet transform based PCA and maximum fusion rule.

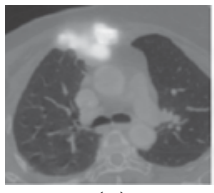

(a)

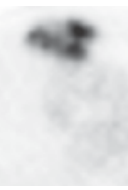

(b)

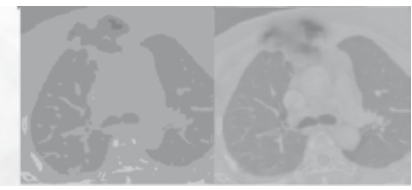

(c) (d)
Figure 4. Result of fused image using Curvelet Transform (a) CT Image (b) PET Image (c) PCA (d) Maximum method

The below Fig.5(a) and fig.5(b) shows CT and PET image. Fig.5(c) shows the multimodal fused image.

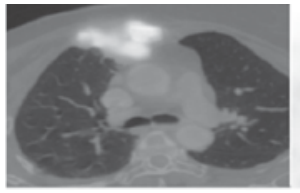

(a)

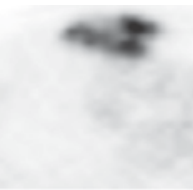

(b)

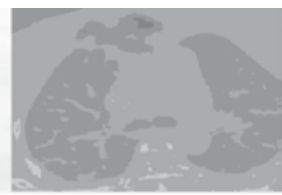

(c)
Figure 5. Result of multimodal image fusion (a) CT Image (b) PET Image (c) Multimodal fusion

\section{Performance Analysis}

The efficiency of the above fusion techniques are determined by the factors such as entropy, Peak Signal to Noise Ratio(PSNR), Structural Similarity Index Measure(SSIM) and Mean Square Error(MSE) [16]. The formula for these metrics are taken from www.mathworks.com[17].

The following Table 1. indicates the performance analysis of the methods included in this work. It is observed from the table that the multimodal image fusion possesses higher values than the rest of the methods with the exception of RMSE value. However, smaller value of RMSE shows better performance of this algorithm. Therefore multimodal image fusion suits well for medical image analysis when compared with other methods.

Table 1. Performance Analysis

\begin{tabular}{|l|l|l|l|l|l|c|}
\hline Transform & Fusion rule & Entropy & PSNR & $\begin{array}{l}\text { Standard } \\
\text { Deviation (SD) }\end{array}$ & $\begin{array}{l}\text { Similarity index } \\
\text { Measure }\end{array}$ & MSE \\
\hline $\begin{array}{l}\text { Discrete } \\
\text { Wavelet }\end{array}$ & Maximum & 5.832 & 25.96 & 34.7321 & 0.421 & 3.256 \\
\cline { 2 - 6 } & PCA & 5.956 & 26.72 & 36.888 & 0.432 & 3.168 \\
\hline Curve let & Maximum & 6.328 & 28.47 & 54.1765 & 0.521 & 2.527 \\
\cline { 2 - 7 } & PCA & 6.786 & 29.56 & 56.192 & 0.556 & 2.496 \\
\hline Multimodal & Maximum and PCA & 7.568 & 49.58 & 60.282 & 0.623 & 0.978 \\
\hline
\end{tabular}




\section{Conclusion}

In this paper, discrete wavelet transform, curvelet transform and multimodal image fusion in UWT and NSCT domain are performed using the fusion rule PCA and maximum method. The output images are displayed and the performance of the above techniques are analyzed by means of entropy, PSNR, SD, SSIM and MSE values. The results indicate that the cascaded combination of UWT and NSCT provides better accuracy with less processing time than the rest of the techniques. Nonetheless, the implementation of these techniques in higher number of input images will decide the accuracy level of the techniques for disease diagnosis. The future work will aim to implement furthermore transform technique in the cascaded network.

\section{References}

1. Medha et al, "Basic medical image fusion methods", International Journal of Advanced Research in Computer Engineering \& Technology, vol 4, issue 3,2015.

2. Deshmukh et al, “A comparative study of different image fusion techniques for toned-mapped images”, International Journal of Scientific \& Engineering Research, vol 7, issue 2, 2016.

3. Govindarajan et al, "Fusion imaging the current trends”, JIMSA, vol 26, no 1,2013.

4. Gattim et al, "Multimodal image fusion using curvelet \& Genetic Algorithm”, Journal of Scientific \& Industrial Research, vol 76, pp-694696,2017.

5. Radhika et al, "Neuro fuzzy logic based fusion algorithm for multimodality images”, ASAR International Conference, ISBN:978-81-927147-45,2013 .

6. Gaurav et al, "Directive contrast based multimodal medical image fusion in NSCT domain”, IEEE Transactions on Multimedia, vol 15, no 5,2013.

7. Arthur et al, "The Non-Subsampled Contourlet Transform theory, design \& application”, IEEE
Transactions on Image Processing, vol 15, no 10, 2006

8. Huda Ahamed et al, "Medical image fusion algorithm based on local average energy motivated PCNN in NSCT domain”, International Journal of Advanced Computer Science \& Applications, vol 7, no 10, 2016.

9. Indira et al, "Performance evaluation of DWT, SWT \& NSCT for fusion of PET \& CT image using different fusion rules”, Biomedical Research, vol 27, issue 1,2016.

10. Anjali Patil et al, "Wavelet Transform based medical image fusion with different fusion methods”, International Journal of Engineering Research \& Applications, vol 5, Issue3,2015.

11. Navneet Kaur et al," Image Fusion based on wavelet \& curvelet transform using ANFIS algorithm”, International Journal of Application or Innovation in Engineering \& Management, vol 3, Issue 9, 2014.

12. Rajashree et al, "Medical image fusion analysis using curvelet transform”, Elsevier Publications,2014.

13. Mamta Sharma, "A review image fusion techniques \&applications”, International Journal of Computer Science \& Information Technologies, vol 7, pp-1082-1085,2016.

14. Jean Luc et al, "The undecimated wavelet decomposition and its reconstruction”, IEEE Transactions on Image Processing, vol 16, no 2, 2007.

15. Parvathi et al, "Medical image fusion using wavelet \& curvelet transform domains", International Journal of Research in Science \& Technology, vol 6, issue 3,2016.

16. Deron et al, "Curvelet based image fusion techniques for medical images”, International Journal for Research in Applied Science \& Engineering Technology, vol3, issue3, 2015.

17. www.mathworks.com accessed for MATLAB code development.

Biographical notes

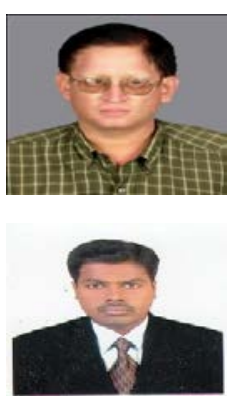

Dr. T R Ganeshbabu. He has obtained his A M I E degree in Electronics and Communication Engineering from The Institution of Engineers (India) in 1993. He received his ME degree in Applied Electronics from Karunya Institute of Technology, Bharathiyar University, Coimbatore, India in 2001. He obtained his PhD from Anna University Chennai, India in 2014 in the field of Medical Image Processing. At present he is a Professor/ECE Muthayammal Engineering College, Rasipuram, Tamilnadu, India. He has authored over 63 research publications in international and national journals and conferences. His special areas of interest are Signal Processing, Wireless Networks, Image processing and Control system. He has been awarded Distinguish Faculty in VIFA 2018 Venus International Foundation Chennai India.

Dr. J. Kirubakaran, Asst. Professor, Department of ECE, Muthayammal Engineering College (Autonomous), Rasipuram, Tamilnadu, India. He completed his Ph.D (Information and Communication Engineering) in Anna University, Chennai, Tamilnadu, India in the year of 2017. His research interest is Wireless Communication,Signal Processing, Image processing, Wireless Sensor Networks, RF Section, Nano Electronics, IoT, etc, 